Article

\title{
Hyperfine Anomalies in Gd and Nd
}

\author{
Jonas R. Persson $\mathbb{D}$
}

Department of Physics, Norwegian University of Science and Technology, NO-7491 Trondheim, Norway; jonas.persson@ntnu.no; Tel.: +47-7355-1127

Received: 25 October 2018; Accepted: 14 November 2018; Published: 15 November 2018

Abstract: The hyperfine anomalies in Gd and Nd have been extracted from experimental hyperfine structure constants. In addition to the values of the hyperfine anomaly, new improved values of the nuclear magnetic dipole moment ratios are derived.

Keywords: hyperfine structure; hyperfine anomaly; gadolinium; neodymium; nuclear magnetic dipole moment

\section{Introduction}

The study of hyperfine structure (hfs) in atoms has provided information about the electromagnetic moments of the nucleus as well as information regarding the properties of the electrons in the atom [1,2]. The magnetic hfs has been used to obtain the so-called differential hyperfine anomaly [3,4] in a number of isotopes. The hyperfine anomaly can be used to obtain information on the distribution of magnetization in the nucleus through the so-called Bohr-Weisskopf effect (BW-effect, $\epsilon_{B W}$ ) [3,5,6]. Bohr and Weisskopf [5] showed in their paper that the magnetic dipole hyperfine interaction constant ( $a$-constant) is smaller for an extended nucleus than for a point nucleus. In addition to the effects of magnetization, the extended charge distribution of the nucleus gives rise to a contribution to the hyperfine anomaly through the so-called Breit-Rosenthal effect (BR-effect, $\epsilon_{B R}$ ) [7-10]. It was shown that isotopic variation, in combination with the different contributions to the hfs from the orbital and spin parts of the magnetization in an extended nucleus, could yield large isotopic deviations from the point nucleus. The reason for this is that s- and $p_{1 / 2}$-electrons have a probability of being inside the nucleus, thus probing the isotopic change in charge distribution as well as the distribution of magnetization. The effects can attain quite large absolute values, but these can only be obtained directly for muonic atoms, where precise calculations of the wavefunctions can be performed [3]. Wavefunctions of highly charged ions must take QED corrections [11] into account, making these more difficult to use. For atoms or singly charged ions, it is more sensible to study differential effects. The differential hyperfine anomaly ${ }^{1} \Delta^{2}$, the difference of the BW- and BR-effects between two isotopes, is normally defined as [3]:

$$
1+{ }^{1} \Delta^{2}=\frac{a^{(1)}}{a^{(2)}} \frac{\mu_{I}^{(2)} / I^{(2)}}{\mu_{I}^{(1)} / I^{(1)}} \approx 1+\epsilon_{B W}^{(1)}-\epsilon_{B W}^{(2)}+\epsilon_{B R}^{(1)}-\epsilon_{B R^{\prime}}^{(2)}
$$

where $\mu_{I}$ is the nuclear magnetic dipole moment, and $I$ the nuclear spin for the isotopes involved.

Using the effective operator formalism [12,13], the hyperfine interaction is divided into three parts, orbital, spin-dipole and contact (spin) interaction, where s-electrons only exhibit the contact (spin) interaction. The hyperfine interaction constants can then be expressed as a linear combination of effective radial parameters $a_{l}^{i j}$ and angular coefficients $k_{l}^{i j}$ for a $l^{N_{S}}$-configuration,

$$
a(J)=k_{l}^{01} a_{l}^{01}+k_{l}^{12} a_{l}^{12}+k_{l}^{10} a_{l}^{10}+k_{s}^{10} a_{s}^{10},
$$


where the indices $(i j)$ stand for the rank in the spin and orbital spaces. Of these, only the contact interactions (10) of $\mathrm{s}$ and $p_{1 / 2}$ electrons contribute to the hyperfine anomaly.

We can thus rewrite the general magnetic dipole hyperfine interaction constant in a simpler form when dealing with hyperfine anomaly:

$$
a=a_{n c}+a_{s}+a_{p}=a_{n c}+k_{s}^{10} a_{s}^{10}+k_{p}^{10} a_{p}^{10}
$$

where $a_{s}$ and $a_{p}$ are the contributions due to the contact interaction of $s$ and $p_{1 / 2}$ electrons, respectively, and $a_{n c}$ is the contribution due to non-contact interactions. The experimentally determined hyperfine anomaly, which is defined with the total magnetic dipole hyperfine interaction constant $a$, should then be rewritten to obtain the relative contributions to the hyperfine anomaly [3]:

$$
{ }^{1} \Delta_{\text {exp }}^{2}={ }^{1} \Delta_{s}^{2} \frac{a_{s}}{a}+{ }^{1} \Delta_{p}^{2} \frac{a_{p}}{a},
$$

where ${ }^{1} \Delta_{s}^{2}$ and ${ }^{1} \Delta_{p}^{2}$ are the hyperfine anomaly for s- and p-electrons, respectively.

By performing an analysis of the hyperfine interaction it is possible to deduce the different contributions to the hyperfine interaction constants, and thus to the hyperfine anomaly. That is, the experimental hyperfine anomaly, which shows a state dependence, can be used to extract the hyperfine anomaly for an s- (or p-) electron, ${ }^{1} \Delta_{s}^{2}$.

It has been shown by Persson [14] that it is possible to extract the hyperfine anomaly without knowing the nuclear magnetic dipole moments, provided you know the contribution of the contact interaction to the hyperfine interaction constant in two atomic states:

$$
\frac{a_{A}^{(1)} / a_{A}^{(2)}}{a_{B}^{(1)} / a_{B}^{(2)}} \approx 1+{ }^{1} \Delta_{s}^{2}\left(\frac{a_{s}^{A}}{a^{A}}-\frac{a_{s}^{B}}{a^{B}}\right),
$$

where $A$ and $B$ are two atomic states in the isotopes 1 and 2. The original suggested use was for radioactive isotopes where the atomic factors, $\left(\frac{a_{s}^{A}}{a^{A}}-\frac{a_{s}^{B}}{a^{B}}\right)$, were calibrated to a known hyperfine anomaly between two stable isotopes [14]. However, it is possible to use this method on stable isotopes where the nuclear magnetic dipole moment is not known with high accuracy. This will be shown by applying the method to Gd and Nd.

\section{Hyperfine Structure}

Over the years, many investigations of the hyperfine structure have been carried out in the rare-earth (4f-shell) region [15], and a vast number of hyperfine interaction constants and isotope shift data have been obtained. One would expect the analysis of the hyperfine interaction in this region to be difficult due to a large number of states. However, one finds that many states are very close to pure LS-coupling, especially the low-lying states [16]. It is therefore relatively easy to perform an analysis. Even if the hyperfine structure has been well studied, the nuclear magnetic dipole moments are not always known with high accuracy [17]. The nuclei in this region are often deformed, leading to large nuclear electric quadrupole moments and drastic changes in mean charge square radius [1,18]. It is therefore interesting to study the hyperfine anomaly, both in stable and unstable isotopes, as this gives an indication of changes in the nuclear magnetization distribution.

\subsection{Hyperfine Structure in $\mathrm{Nd}$}

The hyperfine structure in the Nd atom has been studied with high accuracy by Childs and Goodman [19] and Childs [20], in the $4 f^{4} 6 s^{2}{ }^{5} I_{4-8}$ and $4 f^{4} 5 d 6 s{ }^{7} L_{5-11},{ }^{7} K_{4}$ states using the ABMR and LRDR methods, respectively. These states have also been found to be close to LS-coupling ( $98 \%-100 \%$ pure) $[16,20]$, making an analysis of the hyperfine interaction rather simple. The high accuracy of the hyperfine interaction constants and the fact that an analysis has been performed [20] makes it 
possible to use Equation (5). It is important that the atomic factor $\left(\frac{a_{s}^{A}}{a^{A}}-\frac{a_{s}^{B}}{a^{B}}\right)$ attains a relatively large value, in order to avoid an increased sensitivity to errors [14]. It is therefore important to choose the atomic states in the analysis with great care. From the ratios of the $a$ constants between the two stable isotopes ${ }^{143} \mathrm{Nd}$ and ${ }^{145} \mathrm{Nd}$, we chose the $4 f^{4} 5 d 6 s{ }^{7} L_{5}$ and $4 f^{4} 5 d 6 s{ }^{7} K_{4}$ states for extraction of the hyperfine anomaly as states B in Equation (5), and used $4 f^{4} 5 d 6 s{ }^{7} L_{11}$ as state A. The experimental $a$-constants with ratios and contact contribution are given in Table 1 . The hyperfine anomaly for s-electrons were deduced using Equation (5) and the results are presented in Table 2. The error of the hyperfine anomaly is only due to the experimental errors of the hyperfine interaction constants, as the errors in the contact contribution (i.e., the eigenfunctions) are not known. The error of the hyperfine anomaly was therefore too small and should be larger once the uncertainties of the contact contributions are known. Once the hyperfine anomaly was determined it was possible to use this result as a way of obtaining an improved ratio of the nuclear magnetic dipole moments. The nuclear magnetic dipole moment has been measured by Smith and Unsworth [21] using the ABMR technique, and the ratio is given with the calculated ratio in Table 2. In addition, Halford [22] obtained the ratio of the nuclear magnetic moments using the ENDOR technique, which is also given in Table 2. We also note that the $a$-constants ratio in $4 f^{4} 6 s^{2}{ }^{5} I_{4-8}$ states is the same as the "new" ratio. This is an indication that the hyperfine anomalies in these states are very small or zero. This is not surprising, as the configuration does not contain an unpaired s-electron, thus having no contact interaction. The agreement between the ENDOR measurements and the value obtained here is good taking the errors into account, showing the validity of the method. Using these values, it is possible to determine the hyperfine anomaly in other $\mathrm{Nd}$ isotopes.

Table 1. Hyperfine interaction constants in $\mathrm{Nd}$.

\begin{tabular}{|c|c|c|c|c|}
\hline & $a^{143} N d(M H z)$ & $a^{145} \mathrm{Nd}(\mathrm{MHz})$ & $a^{143} N d / a^{145} N d$ & $a_{s} / a$ \\
\hline $4 f^{4} 5 d 6 s{ }^{7} L_{11}$ & $-151.318(1)^{1}$ & $-93.982(1)^{1}$ & $1.610074(20)$ & $0.45176^{2}$ \\
\hline $4 f^{4} 5 d 6 s{ }^{7} L_{5}$ & $-55.216(1)^{1}$ & $-34.482(1)^{1}$ & $1.601299(55)$ & $-2.26981^{2}$ \\
\hline $4 f^{4} 5 d 6 s{ }^{7} K_{4}$ & $-46.805(1)^{1}$ & $-29.289(1)^{1}$ & $1.598040(65)$ & $-3.21333^{2}$ \\
\hline
\end{tabular}

Table 2. Hyperfine anomaly (hfa) and magnetic dipole moment ratios in $\mathrm{Nd}$.

\begin{tabular}{|c|c|c|}
\hline & ${ }^{143} \Delta_{s}^{145}(\%)$ & $\mu_{I}{ }^{143} N d / \mu_{I}{ }^{145} N d$ \\
\hline $4 f^{4} 5 d 6 s{ }^{7} L_{11}$ & & $1.60860(3)^{1}$ \\
\hline $4 f^{4} 5 d 6 s^{7} L_{5}$ & $0.2013(57)$ & $1.60871(6)^{1}$ \\
\hline $4 f^{4} 5 d 6 s^{7} K_{4}$ & $0.2055(68)$ & $1.60853(7)^{1}$ \\
\hline mean & $0.2034(43)$ & $1.60861(6)^{1}$ \\
\hline$A B M R[21]$ & & $1.626(12)$ \\
\hline ENDOR [22] & & $1.60883(4)$ \\
\hline $4 f^{4} 6 s^{2}{ }^{5} I($ mean $)[19]$ & & $1.60861(2)^{2}$ \\
\hline
\end{tabular}

\subsection{Hyperfine Anomaly in $\mathrm{Gd}$}

Precise studies of the hyperfine structure in Gd have been performed by Unsworth [23], who measured the ${ }^{9} D$ term in the $4 f^{7} 5 d 6 s^{2}$ configuration, and Childs [24], who studied the $4 f^{7} 5 d^{2} 6 s{ }^{11} F$ term. Unsworth [23] found that the contact interaction was very small, indicating that no core-polarisation is present in the $4 f^{7} 5 d 6 s^{2}$ configuration. The configuration does not have an un-paired s-electron should the $4 f^{7} 5 d 6 s^{2}{ }^{9} D$ states not exhibit a hyperfine anomaly, something that can also be seen from the lack of state dependence of the $a$ constant ratios for the two isotopes ${ }^{155,157} \mathrm{G} d$. The levels in the $4 f^{7} 5 d^{2} 6 s{ }^{11} F$ term are reported to be $98 \%-99 \%$ pure L-S coupled states [16], making it possible to use pure L-S coupling in the analysis of the hyperfine interaction [24]. In Table 3 the $a$ constants for the ${ }^{11} F_{2,3,8}$ states are given together with the s-electron contact contribution. The 
difference in contact contribution between the states were smaller compared to $\mathrm{Nd}$, but were sufficient to extract the hyperfine anomaly. It was possible to derive the hyperfine anomaly using Equation (5), and the result is given in Table 4. The ratio of the nuclear magnetic dipole moments when correcting for the hyperfine anomaly was also extracted. The ratio obtained compares well with the ratio of the $4 f^{7} 5 d 6 s^{2}{ }^{9} \mathrm{D}$ states, indicating (as expected) no hyperfine anomaly in these states. The ratio is also in agreement with the ENDOR measurement by Baker et al. [25], but the experimental error is one order of magnitude larger than the derived error. Baker et al. [25] were also able to deduce the hyperfine anomaly of $\mathrm{Gd}^{3}+$ in $\mathrm{CeO}_{3}$ crystals. They obtained a value of ${ }^{157} \Delta_{s}^{155}=0.07(12) \%$, in agreement with the present result.

Table 3. Hyperfine interaction constants in Gd.

\begin{tabular}{|c|c|c|c|c|}
\hline & $a^{157} G d(M H z)$ & $a^{155} G d(M H z)$ & $a^{157} G d / a^{155} G d$ & $a_{s} / a$ \\
\hline $4 f^{7} 5 d^{2} 6 s^{11} F_{2}$ & $-227.108(2)^{1}$ & $-172.942(2)^{1}$ & $0.76150(5)$ & $1.2294(36)^{2}$ \\
\hline $4 f^{7} 5 d^{2} 6 s{ }^{11} F_{3}$ & $-161.933(2)^{1}$ & $-123.333(2)^{1}$ & $0.76116(5)$ & $1.0776(32)^{2}$ \\
\hline $4 f^{7} 5 d^{2} 6 s{ }^{11} F_{8}$ & $-106.124(2)^{1}$ & $-80.849(2)^{1}$ & $0.76184(5)$ & $0.8222(25)^{2}$ \\
\hline
\end{tabular}

Table 4. Hyperfine anomaly and magnetic dipole moment ratios in Gd.

\begin{tabular}{|c|c|c|}
\hline & ${ }^{157} \Delta_{s}^{155}(\%)$ & $\mu_{I}{ }^{155} G d / \mu_{I}{ }^{157} G d$ \\
\hline $4 f^{7} 5 d^{2} 6 s^{11} F_{2}$ & $0.108(18)$ & $0.76249(24)^{1}$ \\
\hline $4 f^{7} 5 d^{2} 6 s{ }^{11} F_{3}$ & $0.104(28)$ & $0.76250(24)^{1}$ \\
\hline $4 f^{7} 5 d^{2} 6 s{ }^{11} F_{8}$ & & $0.76250(24)^{1}$ \\
\hline mean & $0.106(16)$ & $0.76250(16)^{1}$ \\
\hline ENDOR [25] & & $0.7633(45)$ \\
\hline $4 f^{7} 5 d 6 s^{2}{ }^{9} D($ mean $)[23]$ & & $0.76254(40)^{2}$ \\
\hline
\end{tabular}

\section{Conclusions}

The method of deriving hyperfine anomaly without knowing the nuclear magnetic moments [14] was applied to ${ }^{155,157} \mathrm{Gd}$ and ${ }^{143,145} \mathrm{Nd}$, giving the first precise values of the hyperfine anomaly for these isotopes. With values of the hyperfine anomaly in elements close to the well-studied Eu, it is possible to study any general trends in the rare-earth region.

In addition, more precise values of the nuclear magnetic dipole moment ratios were obtained. These values are in agreement with the $a$-constant ratios for the ground terms $4 f^{7} 5 d 6 s^{2}{ }^{9} D(G d)$ and $4 f^{4} 6 s^{2}{ }^{5} \mathrm{I}(\mathrm{Nd})$, proving that the hyperfine anomalies in these states are zero or negligible, and that the electron core polarization in the ground terms is very small. Using this method makes it possible to obtain values of the nuclear magnetic dipole moment ratio as well as a value of the hyperfine anomaly solely from hyperfine interaction constants. This is of special interest in the case of systematic studies over long chains of isotopes, in order to obtain information on nuclear structure.

Funding: This research received no external funding.

Conflicts of Interest: The author declares no conflict of interest.

\section{References}

1. Otten, E.W. Treatise on Heavy-Ion Science; Bromely, D.A., Ed.; Plenum: New York, NY, USA, 1989; Volume 8, p. 515.

2. Lindgren, I.; Rosen, A. Case Studies in Atomic Physics; North-Holland Publ. Co.: Amsterdam, The Netherlands, 1974; Volume 4, p. 73.

3. Büttgenbach, S. Magnetic hyperfine anomalies. Hyperfine Interact. 1984, 20, 1-64. [CrossRef] 
4. Persson, J.R. Table of hyperfine anomaly in atomic systems. Atom. Data Nucl. Data Tables 2013, 99, 62-68. [CrossRef]

5. Bohr, A.; Weisskopf, V.F. The influence of nuclear structure on the hyperfine structure of heavy elements. Phys. Rev. 1950, 77, 94. [CrossRef]

6. Fujita, T.; Arima, A. Magnetic hyperfine structure of muonic and electronic atoms. Nucl. Phys. A 1975, 254, 513-541. [CrossRef]

7. Rosenthal, J.E.; Breit, G. The isotope shift in hyperfine structure. Phys. Rev. 1932, 41, 459. [CrossRef]

8. Crawford, M.F.; Schawlow, A.L. Electron-nuclear potential fields from hyperfine structure. Phys. Rev. 1949, 76, 1310. [CrossRef]

9. Ionesco-Pallas, N.J. Nuclear Magnetic Moments from Hyperfine Structure Data. Phys. Rev. 1960, $117,505$. [CrossRef]

10. Rosenberg, H.J.; Stroke, H.H. Effect of a diffuse nuclear charge distribution on the hyperfine-structure interaction. Phys. Rev. A 1972, 5, 1992. [CrossRef]

11. Shabaev, V.M.; Tomaselli, M.; Kühl, T.; Artemyev, A.N.; Yerokhin, V.A. Ground-state hyperfine splitting of high-Z hydrogenlike ions. Phys. Rev. A 1997, 56, 252. [CrossRef]

12. Lindgren, I.; Morrison, J. Atomic Many-Body Theory; Springer Science \& Business Media: Berlin/Heidelberg, Germany, 1986.

13. Sandars, P.G.H.; Beck, J. Relativistic effects in many electron hyperfine structure I. Theory. Proc. R. Soc. Lond. A 1965, 289, 97-107. [CrossRef]

14. Persson, J.R. Extraction of hyperfine anomalies without precise values of the nuclear magnetic dipole moment. Eur. Phys. J. A 1998, 2, 3-4. [CrossRef]

15. Pfeufer, V. On the hyperfine structure in the configurations $4 f^{n} 6 s^{2}, 4 f^{n} 5 d 6 s, 4 f^{n} 6 s 6 p$, and $4 f^{n-1} 5 d 6 s^{2}$ of the lanthanides. Z. Phys. D At. Mol. Clusters 1987, 4, 351-366. [CrossRef]

16. Atomic Energy Levels, the Rare Earth Elements; National Standard Reference Data Series; National Bureau of Standards (U.S.); Circ. No. 60; Martin, W.C., Zalubas, R., Hagen, L., Eds.; U.S. GPO: Washington, DC, USA, 1978.

17. Stone, N.J. Table of nuclear magnetic dipole and electric quadrupole moments. Atom. Data Nucl. Data Tables 2005, 90, 75-176. [CrossRef]

18. Angeli, I.; Marinova, K.P. Table of experimental nuclear ground state charge radii: An update. Atom. Data Nucl. Data Tables 2013, 99, 69-95. [CrossRef]

19. Childs, W.J.; Goodman, L.S. Hyperfine-Structure Constants and g J Values of I 4, 5, 6, 7, 85 Atomic States of Nd 14 3, 14 5. Phys. Rev. A 1972, 6, 1772. [CrossRef]

20. Childs, W.J. Hyperfine structure of high-L states in i 1 43, 145 by atomic-beam laser-rf double resonance. Phys. Rev. A 1991, 44, 760. [CrossRef] [PubMed]

21. Smith, K.F.; Unsworth, P.J. The hyperfine structure of 167Er and magnetic moments of 143, $145 \mathrm{Nd}$ and $167 \mathrm{Er}$ by atomic beam triple magnetic resonance. Proc. Phys. Soc. 1965, 86, 1249. [CrossRef]

22. Halford, D. Electron-Nuclear Double Resonance, Nuclear Moments, and $\left\langle r^{-3}\right\rangle$ of Neodymium-143 (III) and Neodymium-145 (III) in Lanthanum Trichloride. Phys. Rev. 1962, 127, 1940. [CrossRef]

23. Unsworth, P.J. Nuclear dipole, quadrupole and octupole moments of $155 \mathrm{Gd}$ by atomic beam magnetic resonance. J. Phys. B Atom. Mol. Phys. 1969, 2, 122. [CrossRef]

24. Childs, W.J. Hyperfine structure of the $4 f^{7} 5 d^{2} 6 s F_{11}$ term of $155,157 \mathrm{Gd}$ i by laser-rf double resonance. Phys. Rev. A 1989, 39, 4956. [CrossRef]

25. Baker, J.M.; Copland, G.M.; Wanklyn, B.M. Nuclear moments and hyperfine structure anomaly for gadolinium. J. Phys. C Solid State Phys. 1969, 2, 862. [CrossRef]

(C) 2018 by the author. Licensee MDPI, Basel, Switzerland. This article is an open access article distributed under the terms and conditions of the Creative Commons Attribution (CC BY) license (http:/ / creativecommons.org/licenses/by/4.0/). 